\title{
Diabetes enhances the efficacy of AAV2 vectors in the retina: therapeutic effect of AAV2 encoding vasoinhibin and soluble VEGF receptor 1
}

\author{
Nundehui Díaz-Lezama', Zhijian Wu², Elva Adán-Castro' ${ }^{1}$ Edith Arnold', Miguel Vázquez-Membrillo', \\ David Arredondo-Zamarripa ${ }^{1}$, Maria G Ledesma-Colunga', Bibiana Moreno-Carranza', \\ Gonzalo Martinez de la Escalera', Peter Colosi ${ }^{3}$ and Carmen Clapp ${ }^{1}$
}

Adeno-associated virus (AAV) vector-mediated delivery of inhibitors of blood-retinal barrier breakdown (BRBB) offers promise for the treatment of diabetic macular edema. Here, we demonstrated a reversal of blood-retinal barrier pathology mediated by AAV type 2 (AAV2) vectors encoding vasoinhibin or soluble VEGF receptor 1 (sFlt-1) when administered intravitreally to diabetic rats. Efficacy and safety of the AAV2 vasoinhibin vector were tested by monitoring its effect on diabetes-induced changes in the retinal vascular bed and thickness, and in the electroretinogram (ERG). Also, the transduction of AAV2 vectors and expression of AAV2 receptors and co-receptors were compared between the diabetic and the non-diabetic rat retinas. AAV2 vasoinhibin or AAV2 sFlt-1 vectors were injected intravitreally before or after enhanced BRBB due to diabetes induced by streptozotocin. The BRBB was examined by the Evans blue method, the vascular bed by fluorescein angiography, expression of the AAV2 EGFP reporter vector by confocal microscopy, and the AAV2 genome, expression of transgenes, receptors, and co-receptors by quantitative PCR. AAV2 vasoinhibin and sFlt-1 vectors inhibited the diabetes-mediated increase in BRBB when injected after, but not before, diabetes was induced. The AAV2 vasoinhibin vector decreased retinal microvascular abnormalities and the diabetes-induced reduction of the B-wave of the ERG, but it had no effect in non-diabetic controls. Also, retinal thickness was not altered by diabetes or by the AAV2 vasoinhibin vector. The AAV2 genome, vasoinhibin and sFlt-1 transgenes, and EGFP levels were higher in the retinas from diabetic rats and were associated with an elevated expression of AAV2 receptors (syndecan, glypican, and perlecan) and co-receptors (fibroblast growth factor receptor 1, av $\beta 5$ integrin, and hepatocyte growth factor receptor). We conclude that retinal transduction and efficacy of AAV2 vectors are enhanced in diabetes, possibly due to their elevated cell entry. AAV2 vectors encoding vasoinhibin and sFlt-1 may be desirable gene therapeutics to target diabetic retinopathy and macular edema.

Laboratory Investigation (2016) 96, 283-295; doi:10.1038/labinvest.2015.135; published online 16 November 2015

Blood-retinal barrier breakdown (BRBB) occurs in diabetic macular edema (DME), a complication of diabetic retinopathy (DR) where swelling of the central retina causes visual impairment. ${ }^{1}$ The intravitreal delivery of antiangiogenic and anti-vasopermeability factors can be an effective therapy to control DR and DME but often requires repeated treatments, which raise the risk of infection or other ocular complications. ${ }^{1}$ By producing a more sustained therapy, viral vector-mediated gene transfer could avoid repeated intraocular administration and provide a permanent solution.
The most promising ocular gene therapy for $\mathrm{DR}$ is based on the intravitreal delivery of recombinant adeno-associated virus (AAV) vectors. They are generally safe, and the best characterized type 2 serotype (AAV2) produces long-term transgene expression in retinal ganglion cells after intravitreal delivery. ${ }^{2}$ AAV2 vectors encoding inhibitors of vasopermeability such as soluble VEGF receptor 1 (sFlt-1), ${ }^{3}$ vasoinhibin, ${ }^{3}$ angiostatin, ${ }^{4}$ and angiotensin- $(1-7)^{5}$ reduce retinal vascular leakage in diabetic rats after intravitreal injection. In these studies the vectors were supplied before, not after, inducing diabetes. However, testing the efficacy of

\footnotetext{
IInstituto de Neurobiología, Universidad Nacional Autónoma de México, Querétaro, Mexico; ${ }^{2}$ Ocular Gene Therapy Core, National Eye Institute, NIH, Bethesda, MD, USA and ${ }^{3}$ BioMarin Pharmaceutical, Novato, CA, USA

Correspondence: Professor C Clapp, PhD, Instituto de Neurobiología, Universidad Nacional Autónoma de México, Campus UNAM-Juriquilla, Querétaro 76230, México. E-mail: clapp@unam.mx

Received 2 April 2015; revised 16 October 2015; accepted 20 October 2015
} 
gene therapeutics after diabetes is well-established seems more medically relevant than developing prophylactic strategies. Recent studies have also emphasized the risk of systemic vector dissemination and immune activation following high doses of intravitreally delivered vectors, ${ }^{2}$ so enhancing transduction efficiency is a primary goal.

In this study, we evaluated the inhibitory effect of AAV2 vectors encoding vasoinhibins and sFlt- 1 on diabetes-induced BRBB when injected after BRBB is well-established. We found that the AAV2 vectors reverse BRB pathology and protect against retinal microvascular abnormalities and electrophysiological dysfunction; moreover, their retinal transduction is enhanced in the diabetic state by mechanisms that may include the upregulation of AAV2 primary receptors and co-receptors.

\section{MATERIALS AND METHODS}

\section{Production of AAV2 Vectors}

cDNAs encoding vasoinhibins (codons 1-142 of human prolactin), sFlt-1, and enhanced green fluorescent protein (EGFP) were obtained and cloned into AAV2 vectors downstream of a cytomegalovirus (CMV) immediate early promoter as previously described. ${ }^{3}$ AAV2 preparations were produced using a three-plasmid cotransfection system and purified by polyethylene glycol precipitation and cesium chloride density gradient fractionation using a previously described method. ${ }^{6}$ The purified vectors were formulated in $10 \mathrm{mM}$ Tris- $\mathrm{HCl}$ and $180 \mathrm{mM} \mathrm{NaCl}(\mathrm{pH} 7.4)$ and stored at $-70^{\circ} \mathrm{C}$ before use. Quantification of vectors was done by real-time PCR using linearized plasmid standards. The titers of the vector preparations were $1.4 \times 10^{12}$ vector genomes $(\mathrm{vg})$ per ml for AAV2 vasoinhibin and AAV2 sFlt-1, and $1.4 \times 10^{13}$ vg per ml for AAV2 EGFP. Two microliters of vector or vehicle was injected into the vitreous.

\section{Animals}

Male Wistar rats (250 to $300 \mathrm{~g}$ ) were cared for in accordance with the US National Research Council's Guide for the Care and Use of Laboratory Animals (8th edn, National Academy Press, Washington, DC, USA). The Bioethics Committee of the Institute of Neurobiology of the National University of Mexico (UNAM) approved all animal experiments.

\section{Groups without gene therapy}

Animals were injected with a single intraperitoneal (i.p.) dose of streptozotocin (STZ; $60 \mathrm{mg} / \mathrm{kg}$ in $10 \mathrm{mM}$ citrate buffer, $\mathrm{pH}$ 4.5) (Sigma-Aldrich, St Louis, MO) or vehicle after overnight fasting. Rats with a blood glucose concentration $\geq 250 \mathrm{mg} / \mathrm{dl}$ at $48 \mathrm{~h}$ were considered diabetic. Two, 4, and 6 weeks after STZ, rats were anesthetized with $70 \%$ ketamine and 30\% xylazine $(1 \mu \mathrm{l} / \mathrm{g}$ body weight, i.p.), and the BRBB was examined by the Evans blue method.

\section{Groups with gene therapy after diabetes}

Animals were injected with STZ or vehicle following the above procedures; 2 weeks later the diabetic and non-diabetic rats were anesthetized, and AAV2 vector $\left(2.8 \times 10^{9}\right.$ vg per eye $)$ or vehicle was injected into the vitreous as previously described. ${ }^{7}$ Four weeks after vector administration, some of the animals were anesthetized as above, and the BRBB was examined by the Evans blue method; other animals were subjected to fluorescein angiography, or to electroretinogram (ERG) examination and killed by decapitation to analyze retinal thickness. In addition, other rats were exposed to a $\mathrm{CO}_{2}$-saturated inhalation chamber and killed by decapitation; their retinas were analyzed by quantitative PCR (qPCR) or flat-mounted for confocal microscopy evaluation.

\section{Groups with gene therapy before diabetes}

Animals were injected intravitreally with AAV2 vector $\left(2.8 \times 10^{9}\right.$ vg per eye $)$ or vehicle, and 4 weeks later diabetes was induced with STZ. Four weeks post-STZ, retinas were evaluated by the Evans blue method.

In other experiments, retinas from non-diabetic and six-week diabetic rats were processed for qPCR, immunohistochemistry, or dissected, weighed, and homogenized in $150 \mu \mathrm{l}$ lysis buffer $(0.5 \%$ Igepal, $0.1 \%$ SDS, $50 \mathrm{mM}$ Tris, $150 \mathrm{mM} \mathrm{NaCl}, 1 \mu \mathrm{g} / \mathrm{ml}$ aprotinin, and $100 \mu \mathrm{g} / \mathrm{ml} \mathrm{PMSF}$, $\mathrm{pH}$ 7.4). Retinal homogenates were centrifuged (13 000 r.p.m. for $5 \mathrm{~min}$ ), and the protein in the supernatant was evaluated by the Bradford method. ${ }^{8}$

\section{Evans Blue Method}

The BRBB was evaluated by the Evans blue method, as previously reported. ${ }^{9}$ Briefly, anesthetized rats were injected (intrajugularly) with the Evans blue tracer $(45 \mathrm{mg} / \mathrm{kg}$, SigmaAldrich). Two hours later, $1 \mathrm{ml}$ of blood was drawn from the heart to measure the Evans blue concentration in plasma, and the rats were perfused for $2 \mathrm{~min}$ via the left ventricle with PBS $\left(\mathrm{pH} 3.5\right.$ at $\left.37^{\circ} \mathrm{C}\right)$ at a pressure that allowed a flow rate of $70 \mathrm{ml} / \mathrm{min}$ before insertion of catheter and start of perfusion. The retina was dissected and vacuum-dried (SPD 1010 SpeedVac System, ThermoSavant, USA) for $5 \mathrm{~h}$. After weighing the tissue, the Evans blue tracer was extracted by incubating each retina in $100 \mu$ l formamide (Mallinckrodt Baker, Phillipsburg, NJ) for $18 \mathrm{~h}$ at $72^{\circ} \mathrm{C}$. The extract was centrifuged at $300000 \mathrm{~g}$ for $60 \mathrm{~min}$ at $4{ }^{\circ} \mathrm{C}$. Absorbance was measured in the supernatant at $620 \mathrm{~nm}$ using the NanoDrop 1000 spectrophotometer (Thermo Fisher Scientific, Wilmington, DE). The tracer concentration in the extracts was calculated from a standard curve of Evans blue in formamide and normalized to the retina and body weight and to the Evans blue concentration in plasma.

\section{Fluorescein Angiography}

Anesthetized rats were injected intrajugularly with $100 \mathrm{mg} / \mathrm{kg}$ of fluorescein isothiocyanate-labeled dextran $(50 \mathrm{mg} / \mathrm{ml}$, FITC-dextran: MW $2 \times 10^{6} \mathrm{Da}$; Sigma-Aldrich). One hour 
later, the rats were killed, and their retinas were flat-mounted, fixed for $4 \mathrm{~h}$ in freshly prepared $4 \%$ paraformaldehyde in $0.1 \mathrm{M}$ PBS at room temperature, washed with PBS, mounted on glass slides with Vectashield (Vector Laboratories (Burlingame, CA), and coverslipped. Retinal flat-mounts were observed and digitalized with a $\times 4$ objective under a fluorescence microscope (Olympus BX60 with a DP70 Olympus camera). Images were tiled to create a picture of the entire retina. The density of the capillary network was analyzed using the AngioTool image analysis sofware ${ }^{10}$ having fixed settings for vessel diameter (1-5) and intensity (15-22).

\section{Electroretinogram}

Rats were anesthetized after dark adaptation overnight, and pupils were fully dilated with a $0.5 \%$ tropicamide and $0.5 \%$ phenylephrin solution. Flash ERG responses were recorded from both eyes by a silver chloride ring electrode placed on the cornea. Two reference electrodes were positioned subcutaneously near the eyes. The light stimulation included a 1-ms flash with an intensity of $0.9 \mathrm{log} \mathrm{cd} . \mathrm{s} / \mathrm{m}^{2}$ (PS33 Plus PhotoStimulator, GRASS Technologies, Warwick, RI). The bandpass was set at $3 \mathrm{~Hz}$ to $0.3 \mathrm{kHz}$ (P511AC Amplifier, GRASS Technologies). Sixteen responses were averaged. The A-wave amplitude was measured from baseline to the trough of the A-wave, and the B-wave amplitude from the trough of the A-wave to the peak of the B-wave.

\section{Retinal Thickness}

Eyes fixed in $4 \%$ paraformaldehyde for $24 \mathrm{~h}$ at room temperature were then dehydrated and embedded in paraffin. Orientation of the serial sections $(8 \mu \mathrm{m})$ was assured by controlling that the cuttings passed through the optic nerve head and the cutting marker. Sections were stained with hematoxilin and eosin, and the total thickness of the retina and of both the inner and outer nuclear layers were measured at $\times 20$ magnification using the ScanScope Digital Scanner and the Image Scope software (Aperio Technologies, Vista, CA). Images were taken at equivalent retinal eccentricities from the optic nerve head, and three measurements were taken on each retina within $1 \mathrm{~mm}$ of the optic nerve.

\section{Quantitative PCR}

\section{$A A V$ transgene expression}

Total RNA was isolated from frozen retinas using TRIzol reagent (Life Technologies, Carlsbad, CA), and cDNA was synthesized with the High-Capacity cDNA Reverse Transcription Kit (Applied Biosystems, Warrington, UK). PCR products were separated on a $1.2 \%$ agarose gel and visualized using ethidium bromide, or detected and quantified with Maxima SYBR Green/ROX qPCR Master Mix (Thermo Scientific, Auburn, AL) in a $10-\mu \mathrm{l}$ final reaction volume containing template and $0.5 \mathrm{mM}$ of each of the following primer pairs for human vasoinhibin: 5'-CTGCCCGATGC CAGGTGA-3' (sense) and 5'-GAAAGTCTTTTTGATTC ATCTGT-3' (antisense); human sFlt-1: 5'-GACCTGGAGT
TACCCTGATGA-3' (sense) and 5'-ATGGTCCACTCCTTAC ACGAC-3' (antisense); glypican 1: 5'-TGGCGCCTACGGTG GAAATGATGT- $3^{\prime}$ (sense) and 5'-GAGTGGCGGCCGAGG TCTTCTGTC-3' (antisense); syndecan 4: 5'-GAACCATGG CGCCTGTCTGC-3' (sense) and 5'-CCTGGGCTCCTCCGT GTCATCT-3' (antisense); perlecan 1: 5'-CTGCCACCTGA CAGTCGC-3' (sense) and 5'-GCTCTGGCACCTGCAG-3' (antisense); hepatocyte growth factor receptor $c$-Met (hgfr c-Met): 5'-TCGTTCCTTGGGATTATTGC-3' (sense) and $5^{\prime}$-TGTTTTGTTTTGGCACAGGA-3' (antisense); $\alpha v \beta 5$ integrin: $5^{\prime}$-CACCTGAATGAAGCCAATGA-3'(sense) and 5'-TCCATGCAAAATCTCCACAG-3' (antisense); fibroblast growth factor receptor 1 ( $f g f r$ 1): 5'-CAACACCAAACCAA ACCGTA-3' (sense) and 5'-GTTTTTCAACCAGCGCAAAG $-3^{\prime}$ (antisense) and hypoxanthine-guanine phosphoribosyltransferase (hprt): 5'-GACCGGTTCTGTCATGTCG-3' (sense) and $5^{\prime}$-ACCTGGTTCATCATCACTAATCAC-3' (antisense). The amplification conditions were $10 \mathrm{~s}$ at $95^{\circ} \mathrm{C}, 30 \mathrm{~s}$ at each primer pair-specific annealing temperature, and $30 \mathrm{~s}$ at $72{ }^{\circ} \mathrm{C}$ for 40 cycles. The PCR data were analyzed by the $2-\Delta \Delta \mathrm{CT}$ method, and cycle thresholds were normalized to the housekeeping gene hprt to calculate mRNA expression levels.

\section{AAV genome copy number}

Total genomic DNA was isolated from frozen, AAV2-injected control and diabetic rat retinas. A 100-ng sample of genomic DNA was tested for the presence of AAV2 DNA by qPCR using primers binding to the CMV promoter. qPCR for the $28 \mathrm{~S}$ ribosomal DNA was used as loading control for normalization purposes. PCR products were detected and quantified with Maxima SYBR Green /ROX qPCR Master Mix using the following primer pairs for CMV: $5^{\prime}$-TGCC CAGTACATGACCTTAT-3' (sense) and 5'-AATGGGGCGG AGTTGTTAC-3' (antisense); and rat 28S ribosomal DNA: 5'-CAGTACGAATACAGACCG-3' (sense) and 5'-GGCAAC AACACATCATCAG-3' (antisense). The amplification conditions for the CMV were $30 \mathrm{~s}$ at $94^{\circ} \mathrm{C}, 30 \mathrm{~s}$ at $60.5^{\circ} \mathrm{C}$, and $30 \mathrm{~s}$ at $72^{\circ} \mathrm{C}$ for 40 cycles, whereas for $28 \mathrm{~S}$ ribosomal DNA the annealing temperature was $56.5^{\circ} \mathrm{C}$. For absolute quantification, standard curves for the two plasmids were performed using concentrations ranging from $10^{7}-10$ molecules per $\mu \mathrm{l}$ as determined by spectrophotometry.

\section{Histological Evaluation of EGFP}

Eyes, without the lens, were fixed in $4 \%$ paraformaldehyde for $30 \mathrm{~min}$ at room temperature and washed with PBS. The entire retinas were carefully dissected from the eyecups, flatmounted on glass slides by making four radial cuts from the edges to the equator, and coverslipped with mounting media (Vectashield) to directly detect EGFP fluorescence by confocal microscopy (LSM 780, Carl Zeiss MicroImaging $\mathrm{GmbH}$, Göttingen, Germany). An observer blind to the experiment captured and evaluated serial optical z-sections from three different areas throughout the retinal ganglion cell layer neighboring the optic nerve. EGFP fluorescence per area 
a

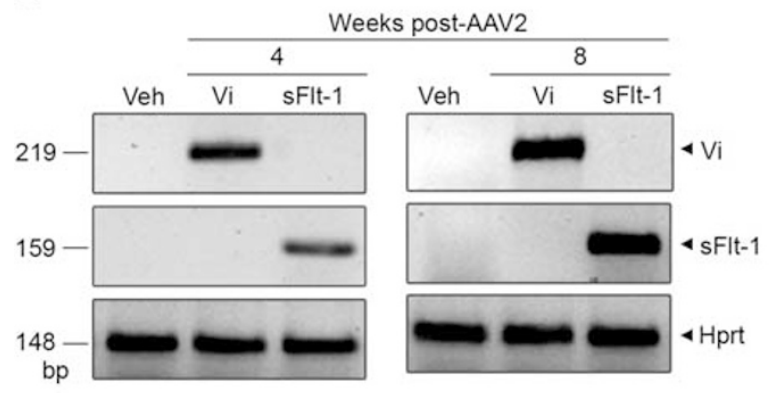

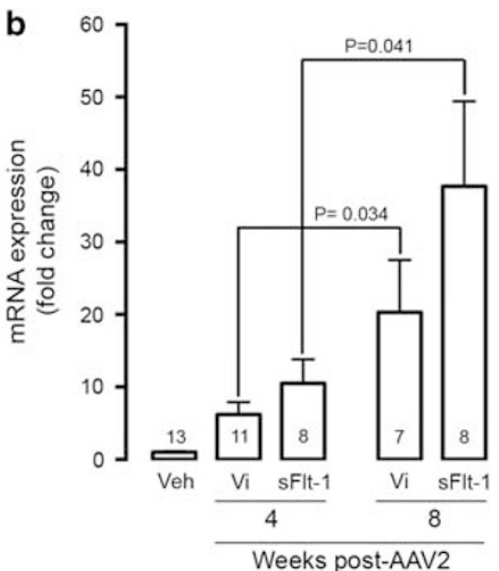

Figure 1 Verification of AAV2-mediated retinal transgene expression. (a) RT-PCR analysis of vasoinhibin (Vi) and sFlt-1 mRNA in retinas obtained from rats 4 and 8 weeks after they had been intravitreally injected with vehicle (Veh), AAV2 Vi, or AAV2 sFlt-1 vectors. The sizes of RT-PCR products are given in bp. Amplification of hypoxanthine-guanine phosphoribosyl transferase (Hprt) was used as an internal standard. (b) qRT-PCR-based quantification of Vi and sFlt-1 mRNA levels in retinas from rats 4 and 8 weeks after they had been intravitreally injected with Veh, AAV2 Vi, or AAV2 sFlt-1 vectors. Bars represent mean \pm s.e.m. Numbers inside bars indicate the number of retinas evaluated. AAV2, adeno-associated virus type 2; qRT-PCR, quantitative reverse transcription PCR.

was quantified using the Pro-Plus image analysis software (Media Cybernetics, Silver Spring, MD).

\section{Immunohistochemistry for FGFR1}

Eyes were fixed in $4 \%$ paraformaldehyde for $4 \mathrm{~h}$ at room temperature and then cryo-protected with 15\% sucrose for 1 day. Subsequently, eyes were frozen in optimal cutting temperature (OCT) compound (Tissue-Tek; Sakura Finetech Torrance, CA), and $12-\mu \mathrm{m}$-thick cryostat sections were mounted on gelatin-coated slides. Sections were washed with PBS to remove OCT, blocked with PBS containing 3\% bovine serum albumin, 5\% normal goat serum, and 0.1\% Triton $\mathrm{X}-100$ for $2 \mathrm{~h}$ at room temperature, and immunostained overnight at $4{ }^{\circ} \mathrm{C}$ with a 1:50 dilution of the anti-FGFR1 monoclonal antibody (ab823 from Abcam PLC, Cambridge, UK). After incubation, samples were washed three times with PBS for $15 \mathrm{~min}$, labeled for $4 \mathrm{~h}$ with a 1:500 dilution of Alexa Fluor 488 goat anti-mouse secondary antibody (Invitrogen, Thermo Fisher Scientific, Waltham, MA), washed three times with PBS, coverslipped using DAPI Vectashield, and imaged by confocal microscopy.

\section{Statistical Analysis}

The statistical analyses were performed using the Sigma Stat 7.0 software (Systat Software, San Jose, CA). Statistical differences between two and more than three groups were determined by the unpaired two-tailed Student's $t$-test and one-way ANOVA followed by Bonferroni's or Tukey's post hoc comparison tests, respectively. The threshold for significance was set at $P<0.05$.

\section{RESULTS \\ Verification of Vasoinhibin and sFlt-1 Transgene Expression in the Retina}

Total retinal RNA from eyes injected with vehicle or with each one of the AAV2 vectors was treated with DNase to eliminate genomic DNA contamination. Amplification of DNasetreated RNA without reverse transcriptase generated no products for vasoinhibin or sFlt-1 (not shown). No human vasoinhibin or human sFlt-1 transcript was present in normal, vehicle-injected eyes (Figure 1a), confirming that the primers do not amplify endogenous vasoinhibin or sFlt-1 in the rat. Products of the expected size for human vasoinhibin and sFlt-1 were amplified in normal retinas 4 and 8 weeks after being transduced with the respective vectors (Figure 1a), and the expression of both transgenes was significantly higher at 8 weeks post transduction (Figure 1b).

\section{The AAV2 Vasoinhibin and AAV2 sFlt-1 Vectors Reverse Diabetes-Induced Blood-Retinal Barrier Pathology}

Table 1 shows the body weight and blood glucose levels for diabetic rats and their matched non-diabetic controls. Diabetic rats showed a reduced weight from 4 to 8 weeks post-STZ treatment, and their blood glucose levels were significantly higher compared with non-diabetic controls at all times.

The STZ rat model of diabetes mimics some of the early changes of human DR, including BRBB. When evaluated using the retinal accumulation of Evans blue or other plasma tracers, BRBB was reported as early as 5 days and up to 10 weeks post-STZ treatment. ${ }^{3,4,9,11-14}$ Here, we confirmed the early and sustained damage of the BRB in this diabetes model by showing that a significant increase in the retinal accumulation of Evans blue-stained albumin occurs at 2 weeks 
Table 1 Body weight and blood glucose concentrations of non-diabetic and diabetic rats

\begin{tabular}{|c|c|c|c|c|}
\hline $\begin{array}{l}\text { Duration of } \\
\text { diabetes (week) }\end{array}$ & Treatment & $n$ & Weight (g) & $\begin{array}{l}\text { Blood glucose } \\
\text { (mg/dl) }\end{array}$ \\
\hline \multirow[t]{2}{*}{2} & $\mathrm{ND}(\mathrm{NI})$ & 4 & $334.5 \pm 8.9$ & $100.0 \pm 3.3$ \\
\hline & $\mathrm{D}(\mathrm{NI})$ & 4 & $325.9 \pm 6.9$ & $478.6 \pm 30.8^{*}$ \\
\hline \multirow[t]{8}{*}{4} & $\mathrm{ND}(\mathrm{NI})$ & 20 & $363.1 \pm 6.3$ & $98.6 \pm 2.5$ \\
\hline & $\mathrm{D}(\mathrm{NI})$ & 20 & $274.4 \pm 9.6^{*}$ & $469.7 \pm 17.9^{*}$ \\
\hline & ND (Veh) & 28 & $341.6 \pm 5.8$ & $103.9 \pm 2.8$ \\
\hline & D (Veh) & 20 & $263.5 \pm 4.5^{*}$ & $486.6 \pm 15.3^{*}$ \\
\hline & ND (Vi) & 9 & $348.9 \pm 7.7$ & $100.6 \pm 5.12$ \\
\hline & $\mathrm{D}(\mathrm{Vi})$ & 3 & $275.9 \pm 6.8^{*}$ & $496.0 \pm 8.5^{*}$ \\
\hline & $\mathrm{ND}(\mathrm{sFlt}-1)$ & 3 & $359.4 \pm 5.9$ & $99.0 \pm 4.8$ \\
\hline & $D($ sFlt-1) & 4 & $285.8 \pm 7.2^{*}$ & $491.3 \pm 19.5^{*}$ \\
\hline \multirow[t]{10}{*}{6} & $\mathrm{ND}(\mathrm{NI})$ & 5 & $442.9 \pm 6.3$ & $105.1 \pm 4.81$ \\
\hline & $\mathrm{D}(\mathrm{NI})$ & 4 & $345.2 \pm 10.4^{*}$ & $464.4 \pm 16.3^{*}$ \\
\hline & ND (Veh) & 16 & $449.9 \pm 6.9$ & $103.8 \pm 3.5$ \\
\hline & D (Veh) & 14 & $327.0 \pm 10.6^{*}$ & $463.3 \pm 11.7^{*}$ \\
\hline & ND (Vi) & 25 & $463.2 \pm 12.9$ & $101.8 \pm 3.3$ \\
\hline & D (Vi) & 25 & $341.1 \pm 11.2^{*}$ & $505.5 \pm 10.4^{*}$ \\
\hline & $\mathrm{ND}(\mathrm{sFlt}-1)$ & 6 & $430.6 \pm 14.6$ & $102.3 \pm 3.5$ \\
\hline & $D(s F \mid t-1)$ & 6 & $319.4 \pm 13.4^{*}$ & $469.2 \pm 18.5^{*}$ \\
\hline & $\mathrm{ND}(\mathrm{EGFP})$ & 10 & $460.7 \pm 9.7$ & $110.5 \pm 4.5$ \\
\hline & D (EGFP) & 11 & $321.0 \pm 21.2^{*}$ & $468.1 \pm 24.9^{*}$ \\
\hline \multirow[t]{2}{*}{8} & ND (Vi) & 7 & $507.2 \pm 8.8$ & $108.9 \pm 4.9$ \\
\hline & D (Vi) & 8 & $370.1 \pm 7.8^{*}$ & $482.2 \pm 16.7^{*}$ \\
\hline
\end{tabular}

Rats treated (D) or not (ND) with streptozotocin (STZ), were non-injected (NI) or injected intravitreally with vehicle (Veh), AAV2 vasoinhibin (Vi), AAV2 sFlt-1 (sFlt-1), or AAV2 EGFP (EGFP) and evaluated at 2, 4, 6, and 8 weeks after STZ. ${ }^{*} P<0.001$ vs respective ND controls. Body weight and blood glucose values were similar within all ND and D groups at the same week period. Values represent mean \pm s.e.m. Number of rats $(n)$.

after STZ injection and is maintained at a similar level during the following 4 and 6 weeks (Figure 2a). To investigate the restoration of the BRB function, vehicle or $2.8 \times 10^{9} \mathrm{vg}$ of the AAV2 vasoinhibin vector or the AAV2 sFlt-1 vector was injected intravitreally into non-diabetic or diabetic rats 2 weeks after treatment with STZ, and the BRBB was quantified 4 weeks after vector administration by the retinal accumulation of Evans blue. As expected, diabetes induced a significant increase in tracer accumulation in vehicle-injected rats (Figure 2b). Treatment with either the AAV2 vasoinhibin vector or the AAV2 sFlt-1 vector reversed the diabetes-induced increase in BRBB. Evans blue accumulation was significantly lower in the diabetic rat retinas transduced with the AAV2 vasoinhibin vector or the AAV2 sFlt-1 vector than in vehicle-injected diabetic rat retinas (Figure 2b). Moreover, diabetic rat retinas transduced with either one of these two vectors showed a level of Evans blue leakage similar to that in vehicle-injected, non-diabetic rats (Figure 2b). The reversal effect is specific to the vasoinhibin and the sFlt-1 transgenes, because the AAV2 EGFP vector had no effect, and none of the vectors modified Evans blue-stained albumin accumulation in non-diabetic rats (Figure 2b). These findings support the therapeutic potential of the AAV2 vasoinhibin and sFlt-1 vectors.

\section{The AAV2 Vasoinhibin Vector Reduces Diabetes-Induced Retinal Microvessel Abnormalities and Electrophysiological Dysfunction}

The therapeutic and safety properties of the AAV2 vasoinhibin vector were further investigated by evaluating the retinal vascular bed, electrophysiological function, and thickness of the retina in rats that had been diabetic for 6 weeks and injected with the vector or with vehicle 2 weeks after diabetes onset (as indicated in Figure 2b). Figure 3a shows representative images of the retinal vasculature of non-diabetic and diabetic rats obtained by fluorescein angiography. Consistent with previous findings using this technique, ${ }^{15,16}$ the retinas of STZ-treated rats showed an intact vascular bed. However, the intensity of the fluorescein signal within the capillary vessels (arrow heads) was reduced in the vehicle-injected diabetic rat compared with the vehicle-injected non-diabetic control (Figure 3a). The fainter capillary vessels in the diabetic condition allowed better visualization of the short vertical capillary segments (indicated by arrows) connecting the superficial and the deep microvascular layers of the inner retina. ${ }^{17}$ Accordingly, the microvessel fluorescein signal per area was significantly reduced in the diabetic rat compared with the non-diabetic control injected with vehicle (Figure 3b). No significant differences were observed in non-diabetic and diabetic rats injected with the AAV2 vasoinhibin vector with respect to the vehicle-injected non-diabetic control.

Next, we used the ERG to evaluate the functional status of the retina. Figure 4a shows representative ERG recordings under scotopic conditions of non-diabetic and diabetic rats injected with vehicle or with the AAV2 vasoinhibin vector. Quantitative analysis showed that the amplitude of the A-wave was similar among all groups (Figure $4 \mathrm{~b}$ ). However, the amplitude of the B-wave decreased significantly in vehicle-injected diabetic rats compared with the vehicleinjected non-diabetic controls (Figure 4c). The reduction of the B-wave was not statistically significant in diabetic rats treated with the AAV2 vasoinhibin vector, and this vector did not modify the B-wave amplitude of non-injected diabetic animals.

Finally, morphological examination of hematoxylin and eosin-stained retinal paraffin sections showed no difference in the thickness of the total retina, the inner nuclear layer, or the outer nuclear layer among groups (Figure $4 \mathrm{~d}$ and e). 
a

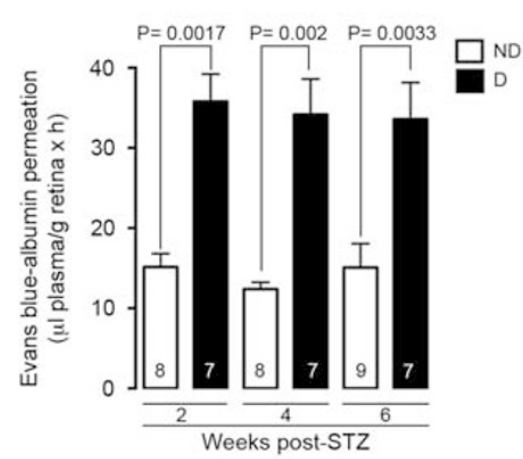

b
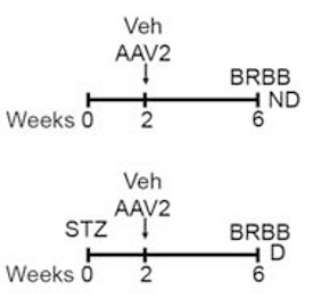

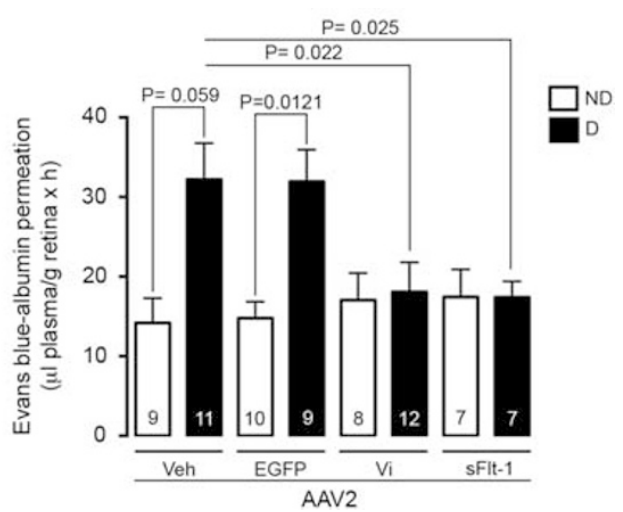

Figure 2 The AAV2 vasoinhibin and AAV2 sFlt-1 vectors reverse diabetes-induced blood-retinal barrier pathology. (a) BRBB evaluated by the retinal accumulation of Evans blue-stained albumin in non-diabetic (ND) control rats and in diabetic (D) rats 2, 4, and 6 weeks after treatment with STZ.

(b) Post-diabetes design diagram: vehicle (Veh), or AAV2 vasoinhibin (Vi), AAV2 sFlt-1, or AAV2-enhanced green fluorescent protein (EGFP) vectors were injected intravitreally into ND or into D rats 2 weeks after treatment with STZ, and BRBB was evaluated 4 weeks after vector administration. Values represent mean \pm s.e.m. Numbers inside bars indicate the number of retinas evaluated. AAV2, adeno-associated virus type 2; BRBB, blood-retinal barrier breakdown; STZ, streptozotocin.

a
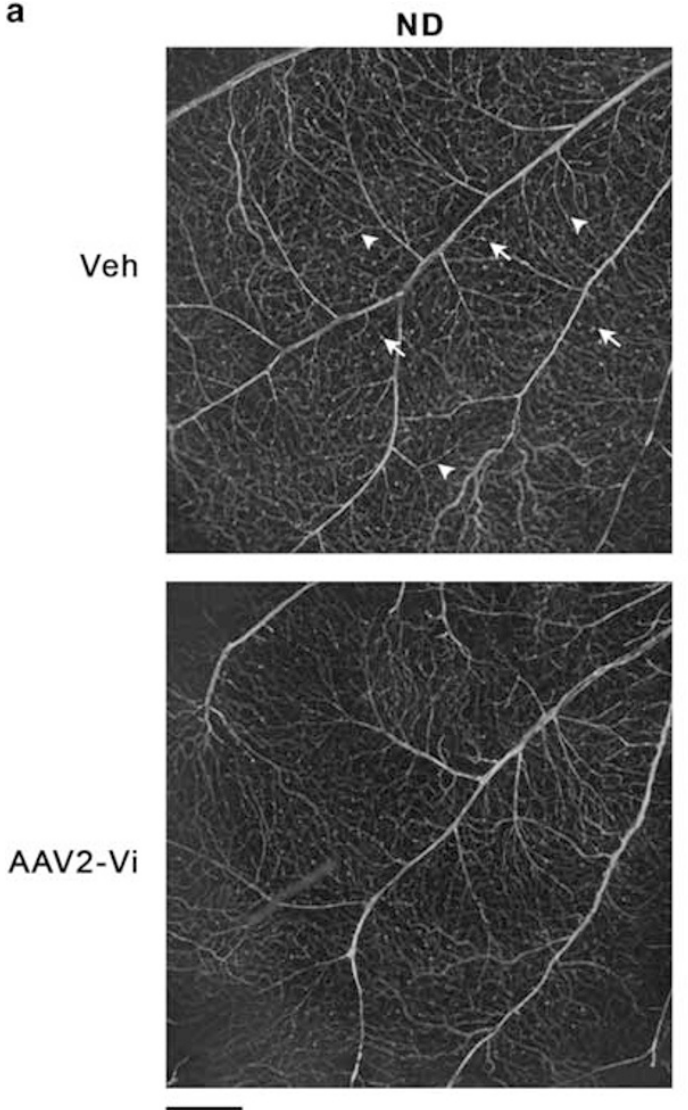
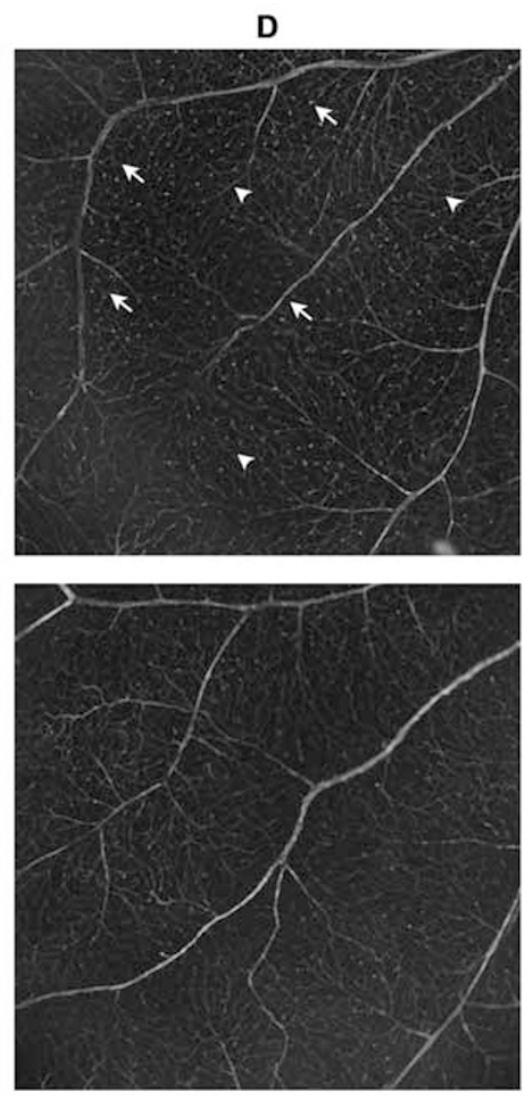

b
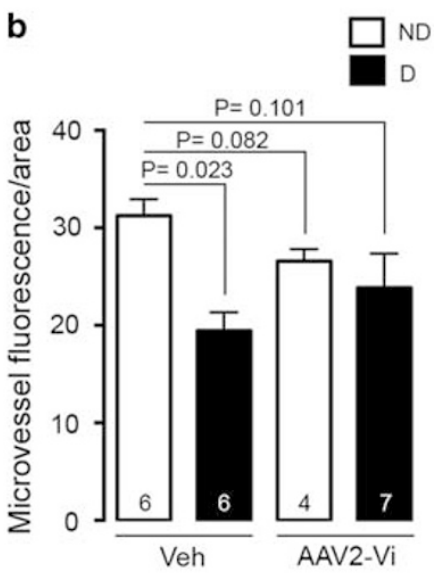

Figure 3 The AAV2 vasoinhibin vector reduces diabetes-induced microvessel abnormalities. (a) Representative images of fluorescein-labeled retinas from non-diabetic (ND) control rats and diabetic (D) rats injected intravitreally with vehicle (Veh) or the AAV2 vasoinhibin (Vi) vector 2 weeks after treatment with STZ and subjected to fluorescein angiography 4 weeks after vector administration. Arrow heads indicate microvessels. Arrows indicate the short vertical capillary segments that connect the superficial and deep microvascular layers of the inner retina. Scale bar, $200 \mu \mathrm{m}$. (b) Quantification of microvessel fluorescein signal per retinal area. Values represent mean \pm s.e.m. Numbers inside bars indicate the number of retinas evaluated. AAV2, adeno-associated virus type 2; STZ, streptozotocin. 
a

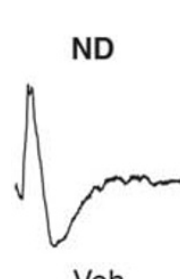

Veh

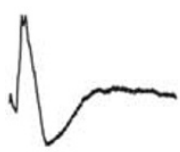

AAV2-Vi
D
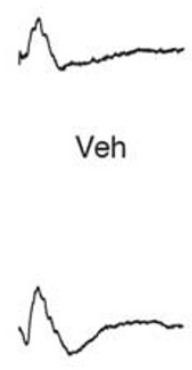

AAV2-Vi b

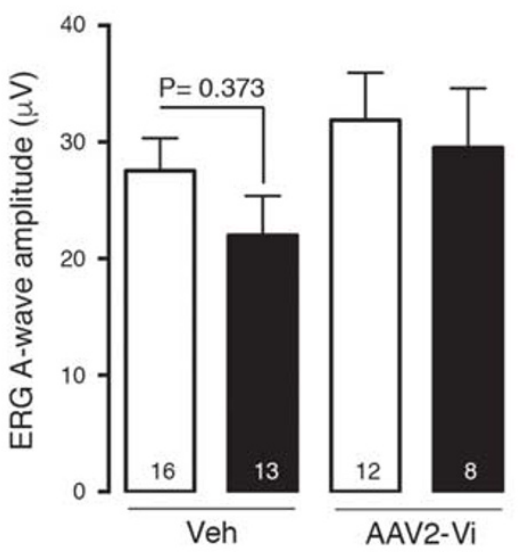

d

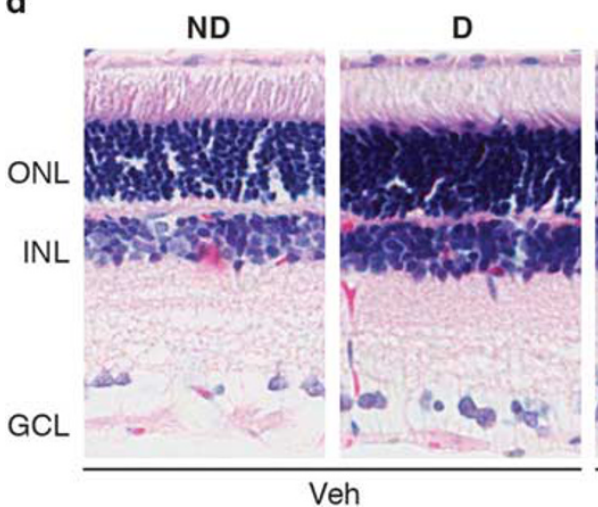

ND

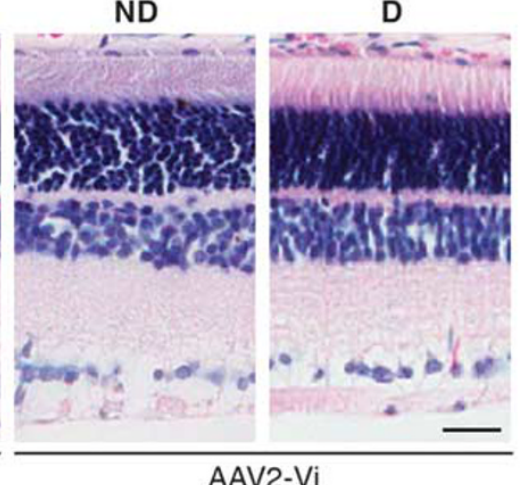

c

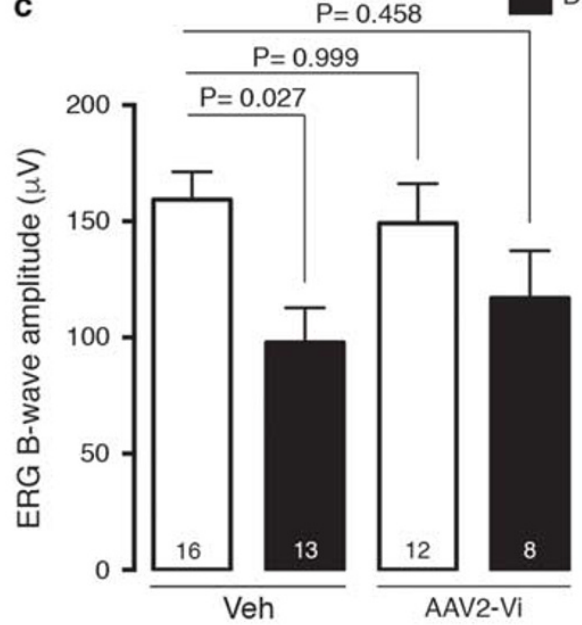

e
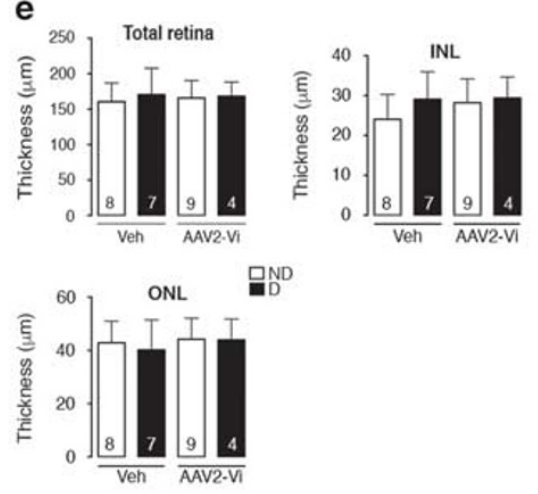

Figure 4 The AAV2 vasoinhibin vector reduces diabetes-induced retinal electrophysiological dysfunction. (a) Representative ERG responses under scotopic conditions of non-diabetic (ND) control rats and diabetic (D) rats injected intravitreally with vehicle (Veh) or the AAV2 vasoinhibin (Vi) vector 2 weeks after treatment with STZ and subjected to ERG evaluation 4 weeks after vector administration. Average amplitudes of A-wave (b) and B-wave (c). (d) Representative paraffin retinal cross-sections stained with hematoxilin and eosin subjected to morphogenic examination of the thickness of total retina and of both the inner nuclear layer (INL) and outer nuclear layer (ONL) (e) of retinas from ND and D rats injected intravitreally with the Veh or the AAV2 Vi 2 weeks after treatment with STZ and subjected to morphometric evaluation 4 weeks after vector administration. Values represent mean \pm s.e.m. Numbers inside bars indicate the number of retinas evaluated. AAV2, adeno-associated virus type 2; ERG, electroretinogram; STZ, streptozotocin.

\section{Diabetes Enhances the Efficacy, Transgene Expression, and Transduction of AAV2 Vectors in the Retina}

The protection against BRBB was reduced or absent when either vector were injected prior to inducing diabetes (Figure 5). In this experiment, vectors were injected intravitreally 4 weeks before inducing diabetes, and BRBB was evaluated 4 weeks thereafter. The increase in BRBB due to diabetes was no longer significant in retinas transduced with the AAV2 vasoinhibin vector, suggesting that vasoinhibins may have a small protective effect. However, the AAV2 sFlt-1 vector did not inhibit diabetes-induced BRBB, and the level of tracer in the vasoinhibin and the sFlt-1-transduced retinas was similar to that observed in the diabetic, vehicle-injected control (Figure 5).

Differences in the experimental design between the beforeand after-diabetes groups do not interfere with the proper evaluation of vector efficacy. The dissimilar times (6 and 4 weeks) following STZ injection result in BRBB of similar magnitudes (Figure 2a). Also, the shorter time post-AAV2 delivery (4 vs 8 weeks) in the after-diabetes group resulted in higher vector efficacy (Figure 2c) even though transgene expression increases over time (Figure 1b). These findings suggest that the diabetic state enhances the efficacy of AAV2 vasoinhibin and AAV2 sFlt-1 vectors to reduce blood-retinal barrier leakage.

To investigate whether increased AAV2 vector efficacy could be due to enhanced transgene expression in the retina of diabetic rats, we compared the expression levels of the vasoinhibin and sFlt-1 mRNAs encoded by the AAV2 vectors, 4 weeks after they had been injected intravitreally into nondiabetic and diabetic rats (Figure 6a). qPCR showed that the retinal expression of the vasoinhibin and sFlt-1 transgenes 

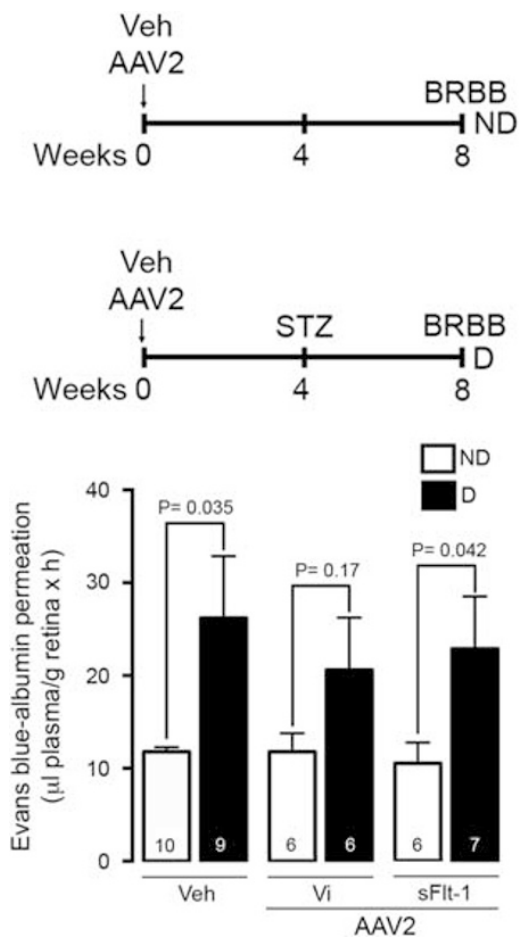

Figure 5 The AAV2 vasoinhibin and AAV2 sFlt-1 vectors fail to reduce diabetes-induced blood-retinal barrier pathology when injected prior to inducing diabetes. Pre-diabetes design diagram: vehicle (Veh), or AAV2 Vi, or AAV2 sFlt-1 vectors were injected intravitreally in non-diabetic rats (ND) or into rats (D) 4 weeks before treatment with STZ, and the retinal permeation of Evans blue-stained albumin was evaluated 8 weeks after vector administration. Values represent mean \pm s.e.m. Numbers inside bars indicate the number of retinas evaluated. AAV2, adeno-associated virus type 2; STZ, streptozotocin.

was nearly fourfold greater in the retinas from diabetic rats than from the non-diabetic controls (Figure 6b). Transgene expression was further investigated by using an AAV2 EGFP reporter vector. The AAV2 EGFP vector was injected intravitreally into non-diabetic rats or into diabetic rats 2 weeks after treatment with STZ, and the levels of EGFP were evaluated by direct fluorescence on flat-mounted retinas 4 weeks after vector delivery. Scattered positive cells and cell fibers were visualized throughout the ganglion cell layer of retinas from non-diabetic rats (Figure 6c). In the diabetic rat retina there was a fivefold enhancement of EGFP fluorescence associated with both somas and projections, and many of the latter corresponded to fibers that make up the optic nerve (Figure 6c).

To investigate whether increased transgene expression involved an enhanced transduction of AAV2 vectors in the retina, we quantified the viral genome copy number by $\mathrm{qPCR}$ at 4 weeks after vectors had been injected intravitreally in non-diabetic and diabetic rats (Figure 6d). The AAV2 genome levels increased significantly in the retina of diabetic rats compared with the non-diabetic controls.

\section{The Expression of Primary Receptors and Co-Receptors of AAV2 Is Elevated in the Retinas from Diabetic Rats}

Transduction efficiency is influenced by receptor- and co-receptor-mediated AAV2 attachment. ${ }^{18}$ To investigate whether a different abundance of AAV2 receptors and co-receptors between the normal and diabetic rat retina might account for the greater recruitment of viral particles in the diabetic state, we evaluated the mRNA levels of AAV2 primary receptors (glypican, syndecan, and perlecan) ${ }^{20}$ and co-receptors (FGFR1, ${ }^{21} \alpha \nu \beta 5$ integrin, ${ }^{22}$ and $\mathrm{HGFR}^{23}$ ) by qPCR in retinas from non-diabetic and 4-week diabetic rats. The expression of all three primary receptors (Figure 7a) and all three co-receptors (Figure 7b) was significantly enhanced in the diabetic rat retina compared with non-diabetic controls. Consistent with these findings, FGFR1 immunoreactivity was faint and diffuse in the photoreceptors outer segments of the retina of non-diabetic rats, whereas it increased dramatically throughout the retina of diabetic rats (Figure 7c). Anti-FGFR1 staining was intense throughout the inner retina (inner nuclear layer, inner plexiform layer, and ganglion cell layer), and less intense and discrete in the outer retina (outer nuclear layer and outer segments) and choroid of diabetic rats. Omission of the primary antibody resulted in no labeling (not shown). Enhanced expression of AAV2 receptors and co-receptors does not reflect a general increase in protein synthesis, since total protein levels were similar in the retinas from diabetic and non-diabetic rats (Figure 7d).

\section{DISCUSSION}

Increased transport through the BRB occurs in DR and DME and is responsible for much of the vision loss due to diabetes. ${ }^{1}$ By producing a sustained expression of antiangiogenic molecules, the AAV vectors eliminate the risks associated with repeated intraocular pharmacotherapy and offer considerable promise for the treatment of DR and DME. Here, we show that diabetes-induced BRB pathology is reversed by the intravitreal delivery of AAV2 vectors encoding the antiangiogenic and anti-vasopermeability factors sFlt-1 and vasoinhibin, and that the AAV2 vasoinhibin vector is a safe procedure for reducing retinal microvessel abnormalities and electrophysiological dysfunction due to diabetes. Moreover, we found that the diabetic state substantially enhances the retinal transduction, transgene expression, and efficacy of AAV2 vectors.

VEGF is a major promoter of BRBB in DR and DME. The levels of VEGF increase in the vitreous of patients with DR and $\mathrm{DME}^{24,25}$ and the clinical use of anti-VEGF molecules improves DME. ${ }^{26,27}$ One such anti-VEGF agent is sFlt-1, which corresponds to the secreted extracellular domain of VEGF receptor 1 and blocks VEGF action by sequestering VEGF. ${ }^{28}$ Vasoinhibins comprise a family of peptides that are generated by the specific proteolytic cleavage of prolactin and they exert potent antiangiogenic and anti-vasopermeability effects. ${ }^{29}$ Vasoinhibins are found in the retina, ${ }^{7}$ and their systemic concentration is reduced in patients with DR. ${ }^{30}$ 
a
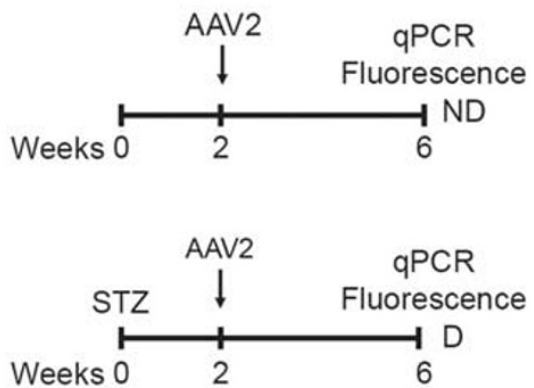

b

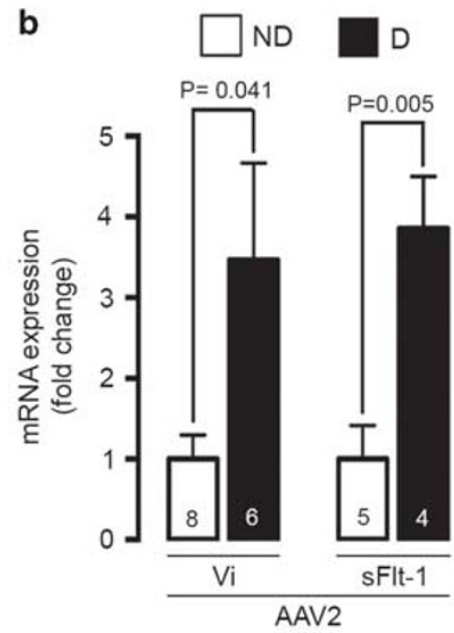

c

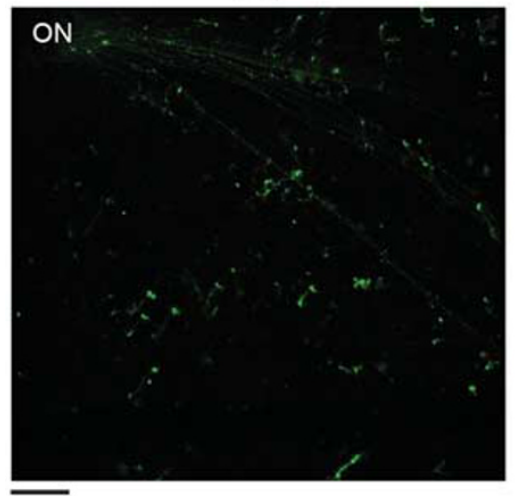

D

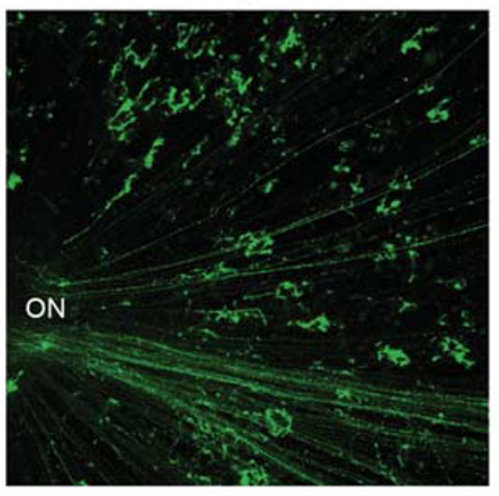

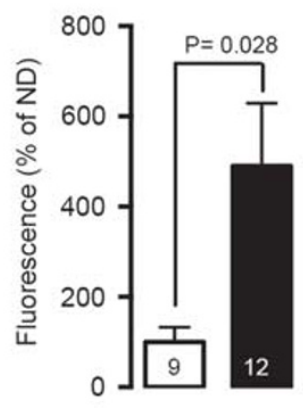

Figure 6 Diabetes enhances the retinal transgene expression and transduction of AAV2 vectors. (a) Post-diabetes design diagram: AAV2 vasoinhibin (Vi), AAV2 sFlt-1, or AAV2 EGFP vectors were injected intravitreally into non-diabetic (ND) or into diabetic (D) rats 2 weeks after treatment with STZ, and the expression of Vi and sFlt-1 mRNA and the levels of the EGFP were evaluated by qPCR and direct fluorescence on flat-mounted retinas, respectively. (b) qRT-PCR-based quantification of Vi and sFlt-1 mRNA levels in retinas from ND and D rats transduced with the AAV2 Vi and AAV2 sFlt-1 vectors. (c) Representative confocal microscope visualization of direct EGFP fluorescence in the ganglion cell layer of a flat-mounted retinal preparation from ND and D rats transduced with the AAV2 EGFP vector. Scale bar, $100 \mu \mathrm{m}$. (d) qPCR-based quantification of AAV genome copy number in retinas from ND and D rats transduced with AAV2 vectors. Values represent mean \pm s.e.m. Numbers inside bars indicate the number of retinas evaluated. AAV2, adeno-associated virus type 2; ON, optic nerve; qRT-PCR, quantitative reverse transcription PCR; STZ, streptozotocin.

Vasoinhibins inhibit ischemia-induced retinal angiogenesis ${ }^{31}$ and reduce diabetes-induced $\mathrm{BRBB}$ by targeting both the inner (vascular endothelial cells) and the outer (retinal pigment epithelium) components of the BRB..$^{32,33}$ The effects of vasoinhibins $s^{3,32,33}$ and sFlt- $1^{34,35}$ against BRBB have been well-documented using Evans blue and a variety of other in vivo and in vitro techniques that have revealed signaling mechanisms mediating their action. For example, VEGF antagonists and vasoinhibins block calcium influx through transient receptor potential channels; they also inhibit the activation of phospholipase $\mathrm{C}$ gamma and phosphatidylinositol-3-kinase-Akt mechanisms, thereby leading to downstream blockage of eNOS activation and the reinforcement of junctional proteins linked to the actin cytoskeleton. ${ }^{32,33,36-38}$
AAV-mediated gene transfer of sFlt-1 successfully reduces BRBB when the vector is delivered before diabetes manifests in the spontaneously diabetic Torii rat. ${ }^{34}$ AAV2 vectors encoding sFlt-1 or vasoinhibin also reduce BRBB in rats when injected before inducing diabetes with STZ. ${ }^{3}$ While these findings demonstrate the preventive effect of these vectors against diabetes-mediated $\mathrm{BRBB}$, the important question of whether BRBB can be reversed by AAV2 vectors had not been addressed. Here, we show that the AAV2 vasoinhibin and sFlt-1 vectors restored the BRB when injected after BRB damage is fully manifest. These findings demonstrate that both vectors reverse a retinal alteration that causes visual impairment in DR and DME.

Furthermore, the AAV2 vasoinhibin vector reduced other aspects of the retinal disease occurring in STZ-treated 
a

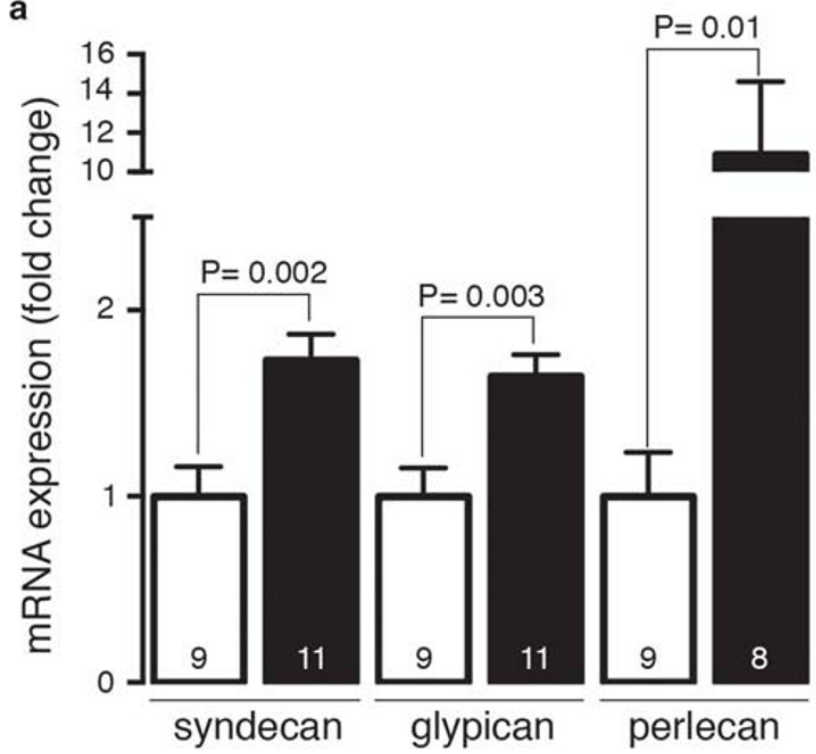

b

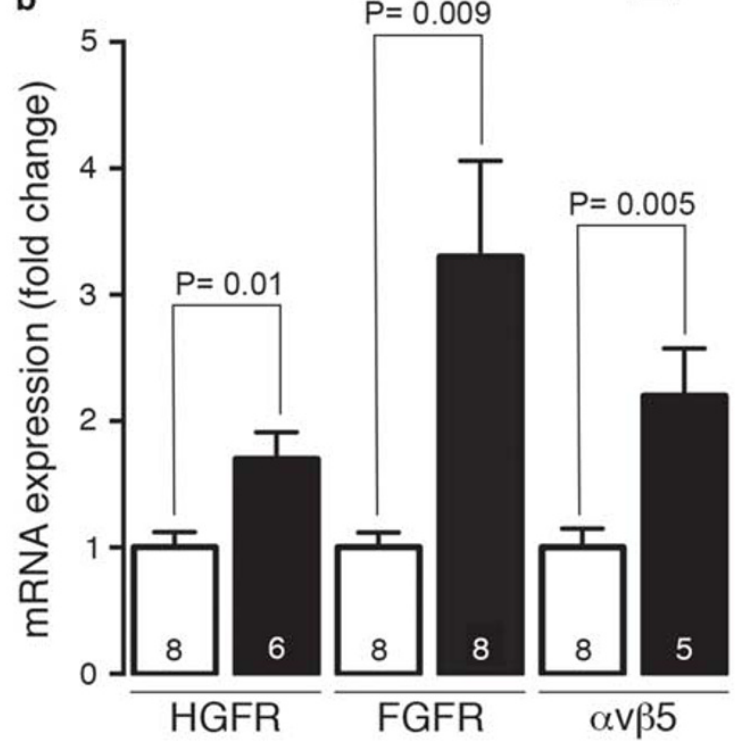

C

ND
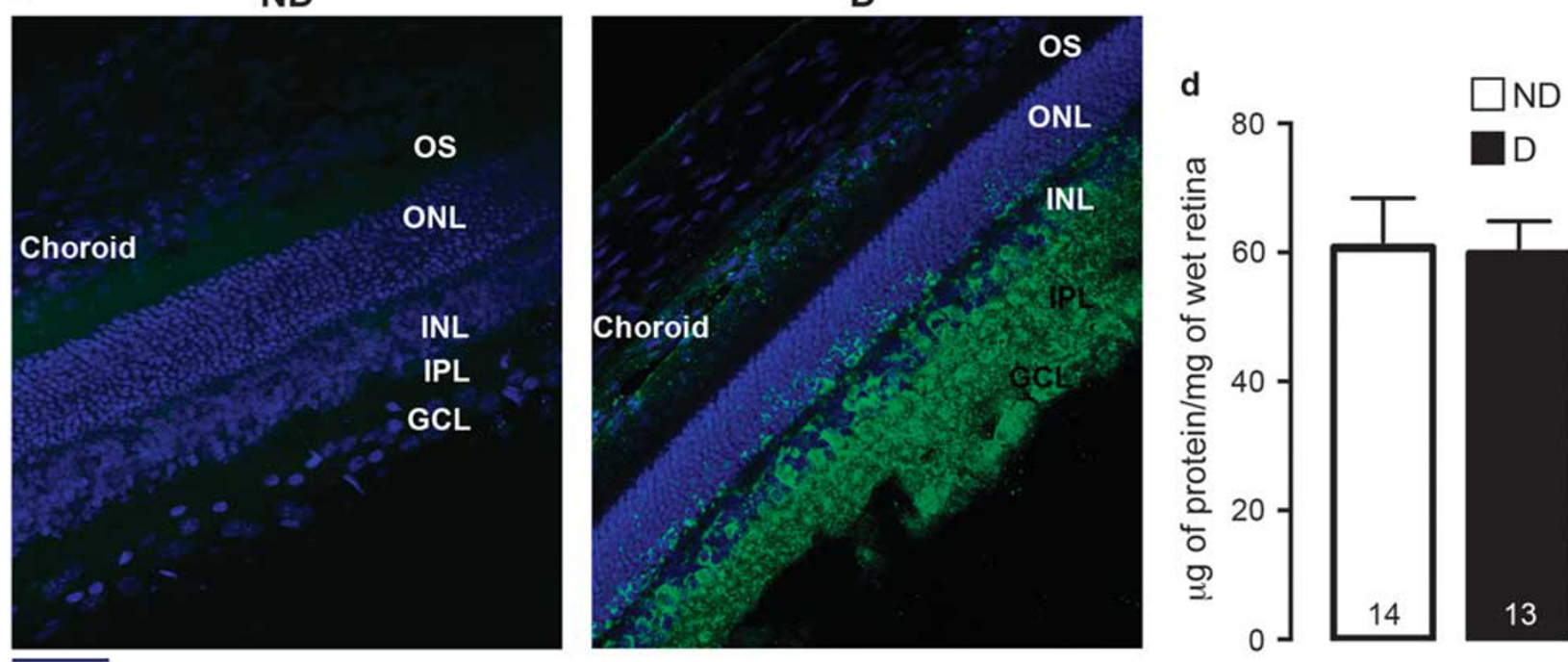

Figure 7 The expression of primary receptors and co-receptors of AAV2 is elevated in the retina from diabetic rats. qRT-PCR-based quantification of AAV2 receptors (glypican, syndecan, and perlecan) (a) and co-receptors (fibroblast growth factor receptor 1 (FGFR), av $\beta 5$ integrin, and hepatocyte growth factor receptor (HGFR)) (b) in retinas from non-diabetic (ND) and 6-week diabetic (D) rats. (c) Immunolocalization and comparison of FGFR1 immunoreactivity between retinal sections of ND and six-week D rats. Scale bar, $50 \mu \mathrm{m}$. (d) Total protein levels in retinas from ND and six-week D rats. Values represent mean \pm s.e.m. Numbers inside bars indicate the number of retinas evaluated. AAV2, adeno-associated virus type 2; GCL, ganglion cell layer; IPL, inner plexiform layer; ONL, outer nuclear layer; OS, outer segments; qRT-PCR, quantitative reverse transcription PCR.

rodents. It inhibited the reduction in fluorescein intensity within capillary vessels that may be caused by factors associated with perfusion abnormalities, such as decreased retinal blood flow, ${ }^{15}$ subnormal retinal oxygenation, ${ }^{39}$ and disruption of the $\mathrm{BRB}^{15}$ (present results). Also, the amplitude of the B-wave, but not that of the A-wave of the ERG, commonly reduced in the STZ rodent model of DR, ${ }^{16,40,41}$ was ameliorated by the AAV2 vasoinhibin vector. Supporting its safety, the vector did not modify the retinal vascular bed and the ERG in non-diabetic rats. Finally, we explored the thinning of the retina which, owing to the neurodegeneration of the inner retina, occurs in diabetic humans, ${ }^{42-45}$ and in some, ${ }^{46-49}$ but not all $^{50}$ diabetic rodent studies. We confirmed that retinal thinning does not occur at 6 weeks post-STZ-treatment, ${ }^{47,49}$ and showed that the AAV2 vasoinhibin vector did not modify it. These observations suggest that the expression of vasoinhibins by AAV2 vectors, as reported for $s \mathrm{Flt}-1,{ }^{51,52}$ is a safe procedure, causing no gross 
retinal changes and protecting against retinal microvascular alterations and loss of retinal electrophysiological function in diabetes.

Reversal of BRB damage by the AAV2 vasoinhibin and sFlt-1 vectors occurred at a dose 10 times smaller than the one used in our previous study $\left(2.8 \times 10^{9}\right.$ vg vs $\left.2.8 \times 10^{10} \mathrm{vg}\right) .^{3}$ However, using this smaller dose and the same experimental parameters of our previous report, ${ }^{3}$ neither of the two vectors clearly prevented $\mathrm{BRBB}$. The absence of effect may relate to the lower dose and/or to the smaller number of animals in our current study. Nonetheless, the vasoinhibin and the sFlt-1 vectors totally reversed the BRBB when delivered after inducing diabetes. Thus, it seemed likely that the efficacy of AAV2 vectors was being potentiated by the diabetic state.

Indeed, transgene expression is enhanced in the diabetic rat retina. The mRNA levels of the vasoinhibin and sFlt-1 transgenes were fourfold higher and the EGFP levels increased fivefold in the retinas from diabetic vs non-diabetic rats. Consistent with the reported tropism of intravitreally delivered AAV2 vectors, ${ }^{53}$ the elevated AAV2 transgene expression occurred in the inner retina, particularly in ganglion cell somas and in their projections that make up the optic nerve. These findings suggest that the diabetic condition potentiates the action of AAV2 vectors by increasing their transduction in permissive cells. Supporting this possibility, we found that AAV2 genome levels were enhanced in the diabetic rat retina compared with the nondiabetic control. Transduction may be facilitated by a higher abundance of AAV2 receptors and co-receptors favoring cell entry.

Heparan sulfate proteoglycans (HSPG) are glycoproteins containing one or more HS chains, and structural determinants of HS interact with AAV2 capsid proteins. ${ }^{54}$ HSPG associated with cell membranes (syndecans and glypicans) serve as primary receptors, enabling vector docking and the subsequent interactions with AAV2 co-receptors (FGFR1, $\alpha v \beta 5$, and HGFR) required for endosomal entry. Also, HSPG associated with the extracellular matrix (perlecans) help store and concentrate AAV2, thus enabling their presentation to attachment and entry receptors. ${ }^{55}$ We found that the mRNA levels of the core proteins in syndecan 4 , glypican 1 , and perlecan 1, and of all three co-receptors (FGFR1, $\alpha v \beta 5$, and HGFR) increased in the diabetic rat retina. Furthermore, FGFR1 immunoreactivity, normally concentrated in photoreceptor cell bodies, ${ }^{56}$ dramatically increased throughout the retina of diabetic rats, mainly in the inner retina, a localization pattern consistent with its reported presence in activated Müller cells. ${ }^{57}$

Increased levels of HSPG and of AAV2 co-receptors are not unexpected in DR and may contribute to its progression. High glucose levels upregulate cell-associated proteoglycans in retinal pericytes ${ }^{58}$ and promote the secretion of HS chains by endothelial cells, ${ }^{59}$ and VEGF increases the retinal expression of the HS side chains of HSPGs. ${ }^{60}$ Perlecan binds FGFs, ${ }^{61,62}$ and the intraretinal stores of immunoreactive bFGF and
HSPG increase in patients with DR. ${ }^{63}$ Of note, abnormal HSPG expression and structure can contribute to vascular leakage and to the release of pro-angiogenic factors in $\mathrm{DR},{ }^{64}$ whereas bFGF, $\alpha v \beta 5$ integrin, and HGF can signal to promote angiogenesis in DR. ${ }^{65}$ However, contrary to what is expected from our findings using rats after 6 weeks of STZ, the mRNA levels of perlecan, the $\left[{ }^{35} \mathrm{~S}\right]$ sulfate incorporation into HSPG, ${ }^{66}$ and the immunolocalization of HSPG $^{67}$ decrease in the retina of diabetic rats at 5 and 11 months after STZ treatment, respectively. These findings suggest that the synthesis of HSPG may change as diabetes progresses and emphasize the need to examine the long-term differential efficacy of AAV2 vectors.

Although the abundance of AAV2 receptors and co-receptors directly correlates with the efficacy of AAV2 vectors, ${ }^{68-70}$ other major events can favor vector entry and transduction in diabetes. Diabetes alters the function and structure of all retinal cell types, ${ }^{71}$ and loss of integrity and changes in retinal architecture are known to increase AAV vector transduction. Disruption of the inner limiting membrane caused by mild proteolytic digestion, ${ }^{72}$ by structural changes of the Müller cell endfeet, ${ }^{73}$ by laser pretreatment, ${ }^{74}$ or by retinal degeneration ${ }^{72}$ allow for enhanced AAV transduction of outer retinal cells after their intravitreal delivery. Moreover, nuclear uptake, capsid uncoating, and second-strand DNA synthesis following receptor binding are major rate-limiting steps in AAV transduction ${ }^{75-78}$ that need to be investigated.

In conclusion, we have demonstrated the therapeutic potential of two AAV2 vectors encoding antivasopermeability and anti-angiogenic factors in DR and DME by delivering them after BRBB is well-established. While intravitreal delivery is a safe treatment, the transduction efficiency of AAV2 vectors can fall short of requirements for adequate retinal transgene expression, ${ }^{68}$ and higher vector doses increase the risk of systemic dissemination and immune activation. $^{2}$ Our demonstration that vector efficacy and transduction after intravitreal delivery are enhanced by the diabetic state provides a way to conquer these problems and emphasizes the advantages of the AAV2 gene transfer approach to treat DR and DME. Non-invasive techniques such as fluorescein angiography, ocular coherence tomography, and magnetic resonance imaging are used increasingly to evaluate BRBB in a wide range of species including mice and rats. ${ }^{79}$ These techniques will help stage AAV2 therapy and follow its efficacy, additional treatment benefits, and side effects over the time period relevant for translational research.

\section{ACKNOWLEDGMENTS}

We thank Fernando López-Barrera, Gabriel Nava, Nydia Hernández-Rios, Estela Albino Sánchez, Michael C Jeziorski, Daniel Mondragón, Antonio Prado, Martín García, and Alejandra Castilla for their technical assistance; and Dorothy D Pless for critically editing the manuscript. Nundehui Diaz-Lezama is a doctoral student from Programa de Doctorado en Ciencias Biomédicas,

Universidad Nacional Autónoma de México (UNAM) and received fellowship 245224 from the National Council of Science and Technology of Mexico 
(CONACYT). Research was supported by CONACYT grants SALUD-2011-1161594 and 247164 to CC.

\section{DISCLOSURE/CONFLICT OF INTEREST}

The authors declare no conflict of interest.

1. Boyer DS, Hopkins JJ, Sorof J et al. Anti-vascular endothelial growth factor therapy for diabetic macular edema. Ther Adv Endocrinol Metab 2013:4:151-169.

2. Vandenberghe $\mathrm{LH}$, Auricchio A. Novel adeno-associated viral vectors for retinal gene therapy. Gene Ther 2012;19:162-168.

3. Ramirez M, Wu Z, Moreno-Carranza B et al. Vasoinhibin gene transfer by adenoassociated virus type 2 protects against Vegf- and diabetesinduced retinal vasopermeability. Invest Ophthalmol Vis Sci 2011;52: 8944-8950.

4. Shyong MP, Lee FL, Kuo PC et al. Reduction of experimental diabetic vascular leakage by delivery of angiostatin with a recombinant adenoassociated virus vector. Mol Vis 2007:13:133-141.

5. Verma A, Shan Z, Lei B et al. Ace2 and Ang-(1-7) confer protection against development of diabetic retinopathy. Mol Ther 2012;20:28-36.

6. Grimm D, Zhou S, Nakai $\mathrm{H}$ et al. Preclinical in vivo evaluation of pseudotyped adeno-associated virus vectors for liver gene therapy. Blood 2003:102:2412-2419.

7. Aranda J, Rivera JC, Jeziorski MC et al. Prolactins are natural inhibitors of angiogenesis in the retina. Invest Ophthalmol Vis Sci 2005;46: 2947-2953.

8. Bradford MM. A rapid and sensitive method for the quantitation of microgram quantities of protein utilizing the principle of protein-dye binding. Anal Biochem 1976:72:248-254.

9. $\mathrm{Xu} \mathrm{Q}$, Qaum T, Adamis AP. Sensitive blood-retinal barrier breakdown quantitation using evans blue. Invest Ophthalmol Vis Sci 2001;42: 789-794.

10. Zudaire E, Gambardella L, Kurcz C et al. A Computational tool for quantitative analysis of vascular networks. PLoS One 2011;6:e27385.

11. Shi $\mathrm{X}$, Liao $\mathrm{S}, \mathrm{Mi} \mathrm{H}$ et al. Hesperidin prevents retinal and plasma abnormalities in streptozotocin-induced diabetic rats. Molecules 2012;17:12868-12881.

12. Zhang J, Wu $Y$, Jin $Y$ et al. Intravitreal injection of erythropoietin protects both retinal vascular and neuronal cells in early diabetes. Invest Ophthalmol Vis Sci 2008;49:732-742.

13. Kusari J, Zhou SX, Padillo E et al. Inhibition of vitreoretinal vegf elevation and blood-retinal barrier breakdown in streptozotocininduced diabetic rats by brimonidine. Invest Ophthalmol Vis Sci 2010;51:1044-1051.

14. Arnold E, Rivera JC, Thebault $\mathrm{S}$ et al. High levels of serum prolactin protect against diabetic retinopathy by increasing ocular vasoinhibins. Diabetes 2010:59:3192-3197.

15. Clermont A, Chilcote TJ, Kita T et al. Plasma kallikrein mediates retinal vascular dysfunction and induces retinal thickening in diabetic rats. Diabetes 2011;60:1590-1598.

16. Kern TS, Tang J, Berkowitz BA. Validation of structural and functional lesions of diabetic retinopathy in mice. Mol Vis 2010;16:2121-2131.

17. Paques $M$, Tadayoni $R$, Sercombe $R$ et al. Structural and hemodynamic analysis of the mouse retinal microcirculation. Invest Ophthalmol Vis Sci 2003;44:4960-4967.

18. Schultz BR, Chamberlain JS. Recombinant adeno-associated virus transduction and integration. Mol Ther 2008;16:1189-1199.

19. Harbison CE, Chiorini JA, Parrish CR. The parvovirus capsid odyssey: from the cell surface to the nucleus. Trends Microbiol 2008;16: 208-214.

20. Summerford C, Samulski RJ. Membrane-associated heparan sulfate proteoglycan is a receptor for adeno-associated virus type 2 virions. J Virol 1998;72:1438-1445.

21. Qing K, Mah C, Hansen J et al. Human fibroblast growth factor receptor 1 is a co-receptor for infection by adeno-associated virus 2 . Nat Med 1999;5:71-77.

22. Summerford C, Bartlett JS, Samulski RJ. Alphavbeta5 integrin: a coreceptor for adeno-associated virus type 2 infection. Nat Med 1999;5: 78-82.

23. Kashiwakura $\mathrm{Y}$, Tamayose $\mathrm{K}$, Iwabuchi $\mathrm{K}$ et al. Hepatocyte growth factor receptor is a coreceptor for adeno-associated virus type 2 infection. J Virol 2005;79:609-614.
24. Aiello LP, Avery RL, Arrigg PG et al. Vascular endothelial growth factor in ocular fluid of patients with diabetic retinopathy and other retinal disorders. N Engl J Med 1994;331:1480-1487.

25. Funatsu $\mathrm{H}$, Yamashita $\mathrm{H}$, Nakamura $\mathrm{S}$ et al. Vitreous levels of pigment epithelium-derived factor and vascular endothelial growth factor are related to diabetic macular edema. Ophthalmology 2006;113: 294-301.

26. Stewart MW. Anti-Vegf therapy for diabetic macular edema. Curr Diab Rep 2014;14:510.

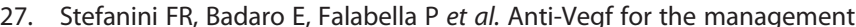
of diabetic macular edema. J Immunol Res 2014;2014:632307.

28. Shibuya M. Vascular endothelial growth factor receptor-1 (Vegfr-1/ Flt-1): a dual regulator for angiogenesis. Angiogenesis 2006;9:225-230.

29. Clapp C, Thebault S, Jeziorski MC et al. Peptide hormone regulation of angiogenesis. Physiol Rev 2009;89:1177-1215.

30. Triebel J, Huefner M, Ramadori G. Investigation of prolactin-related vasoinhibin in sera from patients with diabetic retinopathy. Eur J Endocrinol 2009;161:345-353.

31. Pan $\mathrm{H}$, Nguyen $\mathrm{NQ}$, Yoshida $\mathrm{H}$ et al. Molecular targeting of antiangiogenic factor $16 \mathrm{k} \mathrm{Hprl}$ inhibits oxygen-induced retinopathy in mice. Invest Ophthalmol Vis Sci 2004;45:2413-2419.

32. Garcia C, Aranda J, Arnold E et al. Vasoinhibins prevent retinal vasopermeability associated with diabetic retinopathy in rats via protein phosphatase 2a-dependent Enos inactivation. J Clin Invest 2008;118:2291-2300.

33. Arredondo Zamarripa D, Diaz-Lezama N, Melendez Garcia R et al. Vasoinhibins regulate the inner and outer blood-retinal barrier and limit retinal oxidative stress. Front Cell Neurosci 2014;8:333.

34. Ideno J, Mizukami $\mathrm{H}$, Kakehashi $\mathrm{A}$ et al. Prevention of diabetic retinopathy by intraocular soluble Flt-1 gene transfer in a spontaneously diabetic rat model. Int J Mol Med 2007;19:75-79.

35. Gehlbach $\mathrm{P}$, Demetriades AM, Yamamoto $\mathrm{S}$ et al. Periocular gene transfer of sflt-1 suppresses ocular neovascularization and vascular endothelial growth factor-induced breakdown of the blood-retinal barrier. Hum Gene Ther 2003;14:129-141.

36. Bates DO. Vascular endothelial growth factors and vascular permeability. Cardiovasc Res 2010;87:262-271.

37. Gonzalez C, Corbacho AM, Eiserich JP et al. 16k-prolactin inhibits activation of endothelial nitric oxide synthase, intracellular calcium mobilization, and endothelium-dependent vasorelaxation. Endocrinology 2004;145:5714-5722.

38. Thebault S. Vasoinhibins prevent bradykinin-stimulated endothelial cell proliferation by inactivating Enos via reduction of both intracellular $\mathrm{Ca} 2+$ levels and enos phosphorylation at Ser1179. Pharmaceuticals 2011;4:1052-1069.

39. Luan $\mathrm{H}$, Roberts $\mathrm{R}$, Sniegowski $\mathrm{M}$ et al. Retinal thickness and subnormal retinal oxygenation response in experimental diabetic retinopathy. Invest Ophthalmol Vis Sci 2006;47:320-328.

40. Zhang $Y$, Zhang J, Wang $Q$ et al. Intravitreal injection of exendin-4 analogue protects retinal cells in early diabetic rats. Invest Ophthalmol Vis Sci 2011;52:278-285.

41. Bhatt LK, Addepalli V. Attenuation of diabetic retinopathy by enhanced inhibition of Mmp-2 and Mmp-9 using aspirin and minocycline in streptozotocin-diabetic rats. Am J Transl Res 2010;2:181-189.

42. Biallosterski C, van Velthoven ME, Michels RP et al. Decreased optical coherence tomography-measured pericentral retinal thickness in patients with diabetes mellitus Type 1 with minimal diabetic retinopathy. Br J Ophthalmol 2007;91:1135-1138.

43. van Dijk HW, Kok PH, Garvin $M$ et al. Selective loss of inner retinal layer thickness in type 1 diabetic patients with minimal diabetic retinopathy. Invest Ophthalmol Vis Sci 2009;50:3404-3409.

44. Oshitari T, Hanawa K, Adachi-Usami E. Changes of macular and Rnf thicknesses measured by stratus Oct in patients with early stage diabetes. Eye (Lond) 2009;23:884-889.

45. van Dijk HW, Verbraak FD, Kok PH et al. Decreased retinal ganglion cell layer thickness in patients with type 1 diabetes. Invest Ophthalmol Vis Sci 2010;51:3660-3665.

46. Barber AJ, Lieth E, Khin SA et al. Neural apoptosis in the retina during experimental and human diabetes. early onset and effect of insulin. J Clin Invest 1998;102:783-791.

47. Park SH, Park JW, Park SJ et al. Apoptotic death of photoreceptors in the streptozotocin-induced diabetic rat retina. Diabetologia 2003:46: $1260-1268$. 
48. Berkowitz BA, Roberts R, Stemmler A et al. Impaired apparent ion demand in experimental diabetic retinopathy: correction by lipoic acid. Invest Ophthalmol Vis Sci 2007;48:4753-4758.

49. Martin PM, Roon P, Van Ells TK et al. Death of retinal neurons in streptozotocin-induced diabetic mice. Invest Ophthalmol Vis Sci 2004:45:3330-3336.

50. Feit-Leichman RA, Kinouchi $\mathrm{R}$, Takeda $\mathrm{M}$ et al. Vascular damage in a mouse model of diabetic retinopathy: relation to neuronal and glia changes. Invest Ophthalmol Vis Sci 2005;46:4281-4287.

51. Maclachlan TK, Lukason M, Collins $M$ et al. Preclinical safety evaluation of Aav2-Sflt01- a gene therapy for age-related macular degeneration. Mol Ther 2011;19:326-334.

52. Lukason $\mathrm{M}$, DuFresne $\mathrm{E}$, Rubin $\mathrm{H}$ et al. Inhibition of choroidal neovascularization in a nonhuman primate model by intravitreal administration of an Aav2 vector expressing a novel Anti-Vegf molecule. Mol Ther 2011;19:260-265.

53. Auricchio A, Kobinger G, Anand V et al. Exchange of surface proteins impacts on viral vector cellular specificity and transduction characteristics: the retina as a model. Hum Mol Genet 2001;10: 3075-3081.

54. Kern A, Schmidt K, Leder $C$ et al. Identification of a heparin-binding motif on adeno-associated virus type 2 capsids. J Virol 2003;77: 11072-11081.

55. Vives RR, Lortat-Jacob H, Fender P. Heparan sulphate proteoglycans and viral vectors: ally or foe? Curr Gene Ther 2006;6:35-44.

56. Ozaki S, Radeke MJ, Anderson DH. Rapid upregulation of fibroblast growth factor receptor 1 (Flg) by rat photoreceptor cells after injury. Invest Ophthalmol Vis Sci 2000:41:568-579.

57. Guillonneau X, Regnier-Ricard F, Laplace $\mathrm{O}$ et al. Fibroblast growth factor (Fgf) soluble receptor 1 acts as a natural inhibitor of Fgf2 neurotrophic activity during retinal degeneration. Mol Biol Cell 1998;9: 2785-2802.

58. Fisher EJ, McLennan SV, Yue DK et al. Cell-associated proteoglycans of retinal pericytes and endothelial cells: modulation by glucose and ascorbic acid. Microvasc Res 1994:48:179-189.

59. Han J, Zhang F, Xie J et al. Changes in cultured endothelial cell glycosaminoglycans under hyperglycemic conditions and the effect of insulin and heparin. Cardiovasc Diabetol 2009;8:46.

60. Witmer AN, van den Born J, Vrensen GF et al. Vascular localization of heparan sulfate proteoglycans in retinas of patients with diabetes mellitus and in vegf-induced retinopathy using domain-specific antibodies. Curr Eye Res 2001;22:190-197.

61. Joseph SJ, Ford MD, Barth C et al. A proteoglycan that activates fibroblast growth factors during early neuronal development is a perlecan variant. Development 1996;122:3443-3452.

62. Smith SM, West LA, Hassell JR. The core protein of growth plate perlecan binds Fgf-18 and alters its mitogenic effect on chondrocytes. Arch Biochem Biophys 2007:468:244-251.
63. Hanneken A, de Juan Jr. E , Lutty GA et al. Altered distribution of basic fibroblast growth factor in diabetic retinopathy. Arch Ophthalmol 1991;109:1005-1011.

64. Conde-Knape K. Heparan sulfate proteoglycans in experimental models of diabetes: a role for perlecan in diabetes complications. Diabetes Metab Res Rev 2001;17:412-421.

65. Friedlander $M$, Theesfeld $C L$, Sugita $M$ et al. Involvement of integrins alpha V Beta 3 and alpha $V$ beta 5 in ocular neovascular diseases. Proc Natl Acad Sci USA 1996;93:9764-9769.

66. Bollineni JS, Alluru I, Reddi AS. Heparan sulfate proteoglycan synthesis and its expression are decreased in the retina of diabetic rats. Curr Eye Res 1997;16:127-130.

67. Hammes HP, Weiss A, Hess $S$ et al. Modification of vitronectin by advanced glycation alters functional properties in vitro and in the diabetic retina. Lab Invest 1996:75:325-338.

68. Coura Rdos S, Nardi NB. The state of the art of adeno-associated virusbased vectors in gene therapy. Virol J 2007:4:99.

69. Duan D, Li Q, Kao AW et al. Dynamin is required for recombinan adeno-associated virus type 2 infection. J Virol 1999:73:10371-10376.

70. Duan $D$, Yue $Y$, Yan $Z$ et al. Endosomal processing limits gene transfer to polarized airway epithelia by adeno-associated virus. J Clin Invest 2000;105:1573-1587.

71. Antonetti DA, Barber AJ, Bronson SK et al. Diabetic retinopathy: seeing beyond glucose-induced microvascular disease. Diabetes 2006;55: 2401-2411.

72. Kolstad KD, Dalkara D, Guerin $\mathrm{K}$ et al. Changes in adeno-associated virus-mediated gene delivery in retinal degeneration. Hum Gene Ther 2010:21:571-578.

73. Vacca O, Darche M, Schaffer DV et al. AAV-mediated gene delivery in Dp71-null mouse model with compromised barriers. Glia 2014;62: 468-476.

74. Lee SH, Colosi $\mathrm{P}$, Lee $\mathrm{H}$ et al. Laser photocoagulation enhances adenoassociated viral vector transduction of mouse retina. Hum Gene Ther Methods 2014;25:83-91.

75. Ding $W$, Zhang L, Yan Z et al. Intracellular trafficking of adenoassociated viral vectors. Gene Ther 2005;12:873-880.

76. Campbell EM, Hope TJ. Gene therapy progress and prospects: viral trafficking during infection. Gene Ther 2005;12:1353-1359.

77. Ferrari FK, Samulski T, Shenk T et al. Second-strand synthesis is a ratelimiting step for efficient transduction by recombinant adenoassociated virus vectors. J Virol 1996;70:3227-3234.

78. Thomas $C E$, Storm TA, Huang $Z$ et al. Rapid uncoating of vector genomes is the key to efficient liver transduction with pseudotyped adeno-associated virus vectors. J Virol 2004;78:3110-3122.

79. Berkowitz B. MRI Studies of Blood-Retinal Barrier: New Potential for Translation of Animal Results to Human Application. In: Joussen AM, Gardner TW, Kirchhof B et al. (eds). Retinal Vascular Disease. SpringerVerlag: Berlin Heidelberg, 2007, pp 154-164. 